\title{
La adherencia en los morteros de albañilería
}

JORGE LUIS A. CABRERA, Ing. Investigador de Materiales de Construcción. Centro Técnico para el Desarrollo de los Materiales de Construcción

RESUMEN

Se hace un estudio para determinar la resistencia a la adherencia que alcanzan los morteros de albañileria a los 28 dias. Para ello se prepararon dosificaciones de morteros con 4 tipos de cementos, 2 tipos de arenas y 4 tipos de agregados, lográndose realizar 23 dosificaciones.

Los resultados demuestran la factibilidad de emplear dicho método para determinar la resistencia a la adherencia. Se obtuvieron valores de hasta 0,7 MPa a los 28 dias.

P. Claves: Adherencia, morteros y albañilería.

\section{SUMMARY}

The work presents a study carried out to determine the bond strength reached by bricklaying mortars at 28 days. 23 different dosages were prepared using 4 cement types, 2 sand types and 4 aggregate types.

The results prove the feasibility of usimg the mentioned method to determine bond strength. The values at 28 days reached up to $0.7 \mathrm{MPa}$.

Key words: Bond, mortars, bricklaying.

\section{INTRODUCCIÓN}

La adherencia es una característica propia de los morteros de albañilería y según Menéndez (1), es la propiedad que poseen los morteros de adherirse a los materiales con los cuales están en contacto (piedra, ladrillos, acero, etc.).

Ésta es para muchos autores, investigadores, profesores, constructores y albañiles la propiedad fundamental que deben cumplir los morteros, aunque son pocos los que han realizado un trabajo de investigación o técnico para determinar qué valores de resistencia deben alcanzar los mismos.

Esta falta de mediciones fue lo que dio origen a que se realizara un estudio más profundo para determinar entre que valores oscila la resistencia a la adherenciaen morteros, en dependencia de los materiales con los cuales se está trabajando en la actualidad. Para ello fue necesario estudiar diferentes métodos, así como la efíciencia de los mismos y, por último, confeccionar un gran número de dosificaciones, y ensayos donde se pudieran conjugar variaciones de los componentes de los morteros (cementos, arenas y agregados) y obtener resultados medibles y de confianza.

\section{MATERIALES Y MÉTODOS EMPLEADOS}

\subsection{Materiales}

Cementos: P-350, PP-250, PZ-250 y CA-160

Arenas: Artificial lavada y de mar

Hidrato de cal y DPA

Polvo de piedra

Recebo

Agua

\subsection{Métodos}

Existe algunabibliografiaal respectocomo son los métodos descritos en la norma ASTM 149-66 (2); ensayos de la ETI (3); ensayos del IPTB (4); proyecto de norma de Brasil (5), por Venuat (6) y otros (NBN 813-05; DIN 18 555; MR 21 RILEM 1982; ME-10.IPT, 1981; FE PA 36 LNEC, 1986; Cahier 2669-4 CSTB, 1993 (7).

Todos estos métodos hay que realizarlos con cuidado debido a que como las resistencias que se alcanzan son muy bajas, hace que las dispersiones sean grandes. 
Para el ensayo se prepararon unas losas de hormigón de $300 \times 200 \times 20 \mathrm{~mm}$, las cuales sirven de base al experimento, aunque pudiera ser una pared o cualquier otro tipo de losa en la que sus características sean lo más cercanas a la realidad.

Morteros preparados según (NC 52- 79:93) (8), son adheridos a las losas que se colocan a temperatura ambiente, bajo techo hasta la edad de ensayo.

Un disco de acero de dimensiones predeterminadas conteniendo en su parte superior roscas, es pegado al mortero a ensayar y con una hoja dentada se sierra alrededor del disco de acero hasta llegar a la base del hormigón.

El disco es acoplado a un manómetro, midiendo la fuerza necesaria para separar el disco de la base del hormigón, determinándose de esa forma la fuerza $(F)$, la cual, dividida entre el área del disco (A), nos da la resistencia a la adherencia $\mathrm{R}=\mathrm{F} / \mathrm{A}(\mathrm{MPa})$.

\section{RESULTADOS Y ANÁLISIS}

En la tabla I aparecen los análisis químicos de los 4 tipos de cementos, así como de las dos cales empleadas.

La tabla II muestra los resultados de los ensayos físicos y físico-mecánicos de los cementos. El cemento PP-250 alcanza una resistencia muy alta a los 28 días, esto es normal en estos momentos, por lo que se está trabajando en reajustar los parámetros de producción con respecto a la calidad que debe poseer este cemento.

En la tabla III se muestra el análisis granulométrico de las arenas y agregados en por ciento que pasa.

La tabla IV muestra la adherencia para (en MPA) exhibida por los distintos morteros estudiados en función de la naturaleza de los cementos, arenas y agregados.

Se trabajó con 4 cementos con diferentes características, donde el P-350 es un cemento Portland puro, el PP-250 es un cemento Portland puzolánico, el PZ-250 es un cemento puzolánico y el CA-160 es un cemento de albañilería en base a clinker de cemento portland y caliza.

Las cales son: hidrato de cal de calidad II, según norma(9) es una cal aérea, y el DPA que es una cal obtenida en el proceso de producción del acetileno y que, como puede apreciarse en los resultados, posee buenas características químicas, comparables con el hidrato de cal II.

Como agregado ó árido se emplearon: arena de mar, ésta es una arena fina y lavada para disminuir la cantidad de sales, principalmente cloruros; arena artificial lavada, procedente de las canteras donde es triturada y lavada; el polvo de piedra que, al igual que la arena artificial, se obtiene de la trituración de la piedra $\mathrm{y}$, por último, el recebo, marga calcárea en forma de polvo cuyo contenido de arcilla debe ser menor del $15 \%$ en peso, determinado a través del ensayo del hidrómetro.

TABLA 1

Análisis Químicos en \%

\begin{tabular}{||l|c|c|c|c|c|c||}
\hline \hline Nombre & P-350 & PP-250 & PZ-250 & CA-160 & CAL II & DPA \\
\hline $\mathrm{SiO}_{2}$ & 21,8 & 24,82 & 28,66 & 17,88 & 2,0 & 2,07 \\
\hline $\mathrm{Al}_{2} \mathrm{O}_{3}$ & 5,68 & 6,34 & 4,88 & 5,17 & 1,04 & 1,81 \\
\hline $\mathrm{Fe}_{2} \mathrm{O}_{3}$ & 4,29 & 3,59 & 6,13 & 3,79 & 0,20 & 0,29 \\
\hline $\mathrm{CaO}$ & 60,05 & 53,91 & 49,22 & 56,50 & 67,35 & 68,70 \\
\hline $\mathrm{MgO}$ & 0,91 & 1,56 & 1,21 & 1,21 & --- & -- \\
\hline $\mathrm{SO}_{3}$ & 2,68 & 2,71 & 1,40 & 2,95 & --- & $\cdots$ \\
\hline $\mathrm{PPI}$ & 2,87 & 3,54 & 6,69 & 8,85 & --- & $\cdots$ \\
\hline $\mathrm{RI}$ & 2,66 & 9,82 & 13,26 & 4,97 & --- & --- \\
\hline $\mathrm{Ca}(O H)_{2}$ & --- & --- & -- & -- & 91,01 & 92,84 \\
\hline $\mathrm{CaCO}$ & --- & --- & -- & & 1,20 & 1,64 \\
\hline
\end{tabular}


TABLA \|

Análisis físicos y mecánicos

\begin{tabular}{|c|c|c|c|c|c|c|}
\hline Nombre & & U & $P-350$ & $P P-250$ & $P Z-250$ & CA-160 \\
\hline $\mathrm{FI}$ & & $\min$. & 65 & 70 & 90 & 110 \\
\hline$F F$ & & $\mathrm{~h}: \min$ & $2: 50$ & $3: 10$ & $4: 10$ & $3: 00$ \\
\hline$P E$ & & $\mathrm{~g} / \mathrm{cm}^{3}$ & 3:09 & 3:01 & $2: 80$ & $3: 00$ \\
\hline$S E$ & & $\mathrm{~cm}^{2} / \mathrm{g}$ & 4.278 & 3.687 & 4.867 & 4.129 \\
\hline \multirow[t]{3}{*}{ Rf } & $3 d$ & $\mathrm{MPa}$ & 5,79 & 4,09 & 1,86 & 1,87 \\
\hline & $7 d$ & $\mathrm{MPa}$ & 6,24 & 5,73 & 2,50 & 2,96 \\
\hline & $\underline{28 d}$ & $\mathrm{MPa}$ & 7,29 & 6,32 & 3,94 & 4,17 \\
\hline \multirow[t]{3}{*}{ Rc } & $3 d$ & $\mathrm{MPa}$ & 26,22 & 16,85 & 9,06 & 9,49 \\
\hline & $7 d$ & $\mathrm{MPa}$ & 30,55 & 29,85 & 12,90 & 15,39 \\
\hline & $28 d$ & $\mathrm{MPa}$ & 35,60 & 35,34 & 25,84 & 24,01 \\
\hline
\end{tabular}

TABLA III

Análisis granulométricos en \%

\begin{tabular}{||c|c|c|c|c||}
\hline TAMIZ & A. DE MAR & A. LAVADA & P. PIEDRA & RECEBO \\
\hline 9,52 & 100 & 100 & 100 & 100 \\
\hline 4,76 & 100 & 99,5 & 99,1 & 98,0 \\
\hline 2,38 & 96,1 & 74,1 & 81,8 & 82,0 \\
\hline 1,19 & 93,0 & 38,0 & 58,2 & 69,5 \\
\hline 0,59 & 78,4 & 21,2 & 44,7 & 56,0 \\
\hline 0,297 & 33,1 & 10,4 & 33,0 & 44,1 \\
\hline 0,149 & 5,2 & 4,5 & 22,2 & 25,1 \\
\hline 0,074 & 2,0 & -- & 10,1 & -- \\
\hline
\end{tabular}

Para su utilización en morteros, la arena de mar fue pasada por el tamiz de $2,31 \mathrm{~mm}$; la arena artificial lavada por tamiz $4,76 \mathrm{~mm}$ y el polvo de piedra y el recebo por tamiz de $0,59 \mathrm{~mm}$.

La tabla IV contiene 7 dosificaciones volumétricas, empleándose 4 tipos de cementos de calidades diferentes (tablas I y II), arena de mar y, como agregados, hidrato de cal II y recebo.
$\mathrm{Al}$ aumentar las cantidades de arenas o agregados en las dosificaciones hay siempre una disminución de la resistencia a la adherencia, independientemente del cemento empleado.

Cuando se trabaja con recebo los mejores resultados son con el cemento PP-250 (0,44-0,17 MPa) y los peores con el CA-160 $(0,25-0,06 \mathrm{MPa})$; en las dosificaciones con hidrato de cal, igualmente, las mayores resistencias se 
TABLA IV

\begin{tabular}{|c|c|c|c|c|c|c|c|c|c|c|c|}
\hline \multicolumn{9}{|c|}{ Dosificaciones estudiadas, partes en volumen } & \multirow{2}{*}{\multicolumn{3}{|c|}{ ADHERENCIA, MPa }} \\
\hline \multirow{3}{*}{$\begin{array}{c}N^{\circ} \text { de la } \\
\text { Dosificación }\end{array}$} & \multicolumn{3}{|c|}{ Conglomerantes } & \multicolumn{5}{|c|}{ Áridos } & & & \\
\hline & \multirow{2}{*}{$\begin{array}{c}C \\
P-350\end{array}$} & \multirow{2}{*}{$\begin{array}{l}\text { Hidrato } \\
\text { de Cal }\end{array}$} & \multirow{2}{*}{$\begin{array}{l}\text { Hidrato } \\
\text { de Cal } \\
\text { (D. } \\
\text { acetileno) }\end{array}$} & \multirow{2}{*}{$\begin{array}{l}\text { Propor- } \\
\text { ción }\end{array}$} & \multicolumn{4}{|c|}{ Tipos } & \multirow[b]{2}{*}{$x$} & \multirow[b]{2}{*}{ MIN. } & \multirow[b]{2}{*}{ MAX } \\
\hline & & & & & $\begin{array}{c}\text { Arena de } \\
\text { playa }\end{array}$ & $\begin{array}{c}\text { Arena } \\
\text { artificial }\end{array}$ & $\begin{array}{l}\text { Polvo de } \\
\text { piedra }\end{array}$ & Recebo & & & \\
\hline 1. Morteros de & iento & & & & & & & & & & \\
\hline 1 & 1 & & & 4 & 3 & & & 1 & 0,39 & 0,38 & 0,40 \\
\hline 2 & 1 & & & 6 & 4 & & & 2 & 0,26 & 0,23 & 0,30 \\
\hline 3 & 1 & & & 8 & 5 & & & 3 & 0,10 & 0,10 & 0,10 \\
\hline 4 & 1 & & & 4 & & 3 & 1 & & -- & -- & -- \\
\hline 5 & 1 & & & 5 & & 4 & 1 & & 0,45 & 0,45 & 0,45 \\
\hline 6 & 1 & & & 6 & & 4 & 2 & & 0,39 & 0,37 & 0,40 \\
\hline 7 & 1 & & & 8 & & 6 & 2 & & 0,38 & 0,37 & 0,40 \\
\hline 8 & 1 & & & 4 & & 3 & & 1 & 0,50 & 0,47 & 0,53 \\
\hline 9 & 1 & & & 5 & & 4 & & 1 & 0,46 & 0,42 & 0,50 \\
\hline 10 & 1 & & & 6 & & 4 & & 2 & 0,41 & 0,38 & 0,46 \\
\hline 11 & 1 & & & 8 & & 6 & & 2 & 0,36 & 0,32 & 0,41 \\
\hline
\end{tabular}


TABLA IV (Cont.)

\begin{tabular}{|c|c|c|c|c|c|c|c|c|c|c|c|}
\hline \multicolumn{9}{|c|}{ Dosificaciones estudiadas, partes en volumen } & \multirow{2}{*}{\multicolumn{3}{|c|}{ ADHERENCIA, MPa }} \\
\hline \multirow{3}{*}{$\begin{array}{c}\mathrm{N}^{0} \text { de la } \\
\text { Dosificación }\end{array}$} & \multicolumn{3}{|c|}{ Conglomerantes } & \multicolumn{5}{|c|}{ Áridos } & & & \\
\hline & \multirow{2}{*}{$\underset{P-350}{C}$} & \multirow{2}{*}{$\begin{array}{l}\text { Hidrato } \\
\text { de Cal }\end{array}$} & \multirow{2}{*}{$\begin{array}{l}\text { Hidrato } \\
\text { de Cal } \\
\text { (D. } \\
\text { acetileno) }\end{array}$} & \multirow{2}{*}{$\begin{array}{l}\text { Propor- } \\
\text { ción }\end{array}$} & \multicolumn{4}{|c|}{ Tipos } & \multirow[b]{2}{*}{$x$} & \multirow[b]{2}{*}{ MIN. } & \multirow[b]{2}{*}{ MAX. } \\
\hline & & & & & $\begin{array}{c}\text { Arena de } \\
\text { playa }\end{array}$ & $\begin{array}{c}\text { Arena } \\
\text { artificial }\end{array}$ & $\begin{array}{l}\text { Polvo de } \\
\text { piedra }\end{array}$ & Recebo & & & \\
\hline \multicolumn{12}{|c|}{ 2. Morteros bastardos } \\
\hline 12 & 1 & 0,5 & & 3 & 3 & & & & 0,43 & 0,30 & 0,49 \\
\hline 13 & 1 & 1 & & 4 & 4 & & & & 0,39 & 0,30 & 0,40 \\
\hline 14 & 1 & 2 & & 6 & 6 & & & & 0,14 & 0,10 & 0,17 \\
\hline 15 & 1 & 3 & & 8 & 8 & & & & 0,11 & 0,10 & 0,15 \\
\hline 16 & 1 & 1 & & 3 & & 3 & & & 0,59 & 0,52 & 0,67 \\
\hline 17 & 1 & 1 & & 4 & & 4 & & & 0,49 & 0,45 & 0,52 \\
\hline 18 & 1 & 2 & & 4 & & 4 & & & 0,45 & 0,45 & 0,45 \\
\hline 19 & 1 & 2 & & 6 & & 6 & & & 0,38 & 0,35 & 0,40 \\
\hline 20 & 1 & & 1 & 3 & & 3 & & & 0,52 & 0,52 & 0,52 \\
\hline 21 & 1 & & 1 & 4 & & 4 & & & 0,49 & 0,45 & 0,52 \\
\hline 22 & 1 & & 2 & 4 & & 4 & & & 0,43 & 0,40 & 0,45 \\
\hline 23 & 1 & & 2 & 6 & & 6 & & & 0,36 & 0,30 & 0,40 \\
\hline
\end{tabular}

$\simeq$ 
alcanzan con el cemento PP-250, (0,70-0,07 MPa), seguido por las del cemento P-350, PZ-250, y CA-160 respectivamente, además son ligeramente mayores cuando se emplea el hidrato de cal.

En la tabla IV se muestran 4 dosificaciones diferentes en las cuales se varía la cantidad de arena, en este caso arena artificial lavada y 4 tipos de agregados; la resistencia a la adherencia disminuye al aumentar la cantidad de arena o agregado, y los mejores resultados se obtienen con el hidrato de cal y DPA, seguidos por polvo de piedra y recebo.

Haciendo una comparación respecto a la influencia de los áridos sobre la resistencia a la adherencia, se observa que cuando se emplea arena artificial lavada, la cual es más gruesa, la resistencia es mayor, independientemente del agregado empleado.

\section{CONCLUSIONES}

Por medio de este ensayo se logran obtener resultados de resistenciaa laadherencia para una serie de dosificaciones con diferentes tipos de cementos, arenas y agregados que, aunque presentan alguna dispersión, da una idea de qué valores de resistencia alcanzan los morteros que normalmente se emplean en obras de albañilería.

Los mayores valores de resistencia a la adherencia se obtuvieron cuando se trabajó con la arena artificial lavada como árido y con el hidrato de cal como agregado en general, y los menores con cemento CA-160.

\section{BIBLIOGRAFÍA}

(1) MENÉNDEZ, J.: "Una lección sobre morteros", Folleto. Univ. de la Habana. 1946. 33 p.

(2) C 149-66. Standard Especification for: Bond strenght of mortar to masonry units: Annual book of ASTM Standard. Part 12 (col.) Vig. desde 71-12. pp. 452-463.

(3) Apparatus ETI-RIP-U for testing adhesive strenght: Hungarian Institute for Building Science. 7 p.

(4) PASSOS, J.E.A.: "Evolucao da consolidacao contendo cal hidratada/J.E. Passos; M.A. Cincotto y M.T. de Nobrega. Sao Pablo. Instituto Brasileiro do concreto. 1985. $31 \mathrm{p}$.

(5) Proyecto de norma 02-002.17.003 "Revestimiento de argamasas inorgánicas de paredes e Tetos. Determinación de resistencia de adherencias a tracción", Asociación Brasileira de Normas Técnicas. Río de Janeiro. Brasil. agosto 1993.

(6) VENUAT, M.: “Adherencia (Aditivos y tratamientos de morteros y hormigones)”. Barcelona. Editores Técnicos Asociados. 1972. PP. 67-68.

(7) CARASEK, H. y SELMO, S.: "Método de Ensaio de Determinacao da Resistencia de Aderencia a tracao de Revestimientos de Argamasa". Memorias ENTAC 93, V.1, 17-19 nov. 1993. Sao Paulo. pp. 261-270.

(8) NC52-79:93.: "Morteros de albañilería. Parte 1. Diseño y caracterización”. Código de práctica para la construcción. Vig. enero $1993.8 \mathrm{p}$.

(9) NC54-286:84.: "Hidrato de cal". Especificaciones de calidad. Vig. octubre 1985

NOTA: Se agradece al Dr. César del Olmo las aportaciones realizadas en el presente trabajo. 